\title{
Multi-Agent Zero-Sum Differential Graphical Games for Disturbance Rejection in Distributed Control
}

\author{
Qiang Jiao ${ }^{* a}$, Hamidreza Modares ${ }^{\mathrm{b}}$, Shengyuan Xu ${ }^{\mathrm{a}}$, Frank L. Lewis ${ }^{\mathrm{b}}$, \\ Kyriakos G. Vamvoudakis ${ }^{c}$
}

\author{
${ }^{*}$ Corresponding author. Phone +8613813859536 \\ ${ }^{a}$ School of Automation, Nanjing University of Science and Technology, Nanjing, 210094, Jiangsu, P.R. China, qjiao0312@gmail.com, \\ syxu@njust.edu.cn \\ ${ }^{b}$ University of Texas at Arlington Research Institute, 7300 Jack Newell Blvd. S., Ft. Worth, TX 76118, USA, modares@uta.edu, lew- \\ is@uta.edu \\ ${ }^{c}$ Center for Control, Dynamical-systems and Computation(CCDC), University of California, Santa Barbara, CA 93106-9560,USA, \\ kyriakos@ece.ucsb.edu
}

\begin{abstract}
This paper addresses distributed optimal tracking control of multi-agent linear systems subject to external disturbances. The concept of differential game theory is utilized to formulate this distributed control problem into a multi-player zero-sum differential graphical game, which provides a new perspective on distributed tracking of multiple agents influenced by disturbances. In the presented differential graphical game, the dynamics and performance indices for each node depend on local neighbor information and disturbances. It is shown that the solution to the multi-agent differential graphical games in the presence of disturbances requires the solution to coupled Hamilton-Jacobi-Isaacs (HJI) equations. Multi-agent learning policy iteration (PI) algorithm is provided to find the solution to these coupled HJI equations and its convergence is proven. It is also shown that $\mathrm{L}_{2}-$ bounded synchronization errors can be guaranteed using this technique. An online PI algorithm is given to solve the zero-sum game in real time. A simulation example is provided to show the effectiveness of the online approach.
\end{abstract}

Key words: Graphical games, Hamilton-Jacobi-Isaacs equations, $\mathrm{L}_{2}$-gain, External disturbances, Multi-agent system

\section{Introduction}

The distributed control of multi-agent systems has received much attention in the past years, due to its potential applications in a variety of engineering systems. A rich body of literature has been developed on distributed control methods for consensus and synchronization (Fax \& Murray, 2004; Jadbabaie, Lin \& Morse, 2003; Olfati-Saber \& Murray, 2004; Qu, 2009; Ren \& beard, 2005, 2008; Ren, Beard, \& Atkins, 2005; Tsitsiklis, 1984), and some good surveys can be found in (Lewis et al., 2014; Qu, 2009; Ren, Beard, \& Atkins, 2005).

The objective in distributed control is to design for each agent a control protocol, depending only on local neighbor information, to guarantee synchronized behavior of all the agents by reaching agreement on certain quantities of interests. Although agent dynamics may be subject to external disturbances, most of the existing distributed control methods ignore these disturbances. However, to avoid performance degradation, it is necessary to consider the effect of disturbances in the distributed control problem formulation a priori.

Recently, disturbance attenuation has been taken into account in distributed control of multi-agent systems. Lin,
Jia, and Li (2008) studied the $H_{\infty}$ consensus problem for first-order dynamical systems. Li, Duan \& Huang (2009) transformed the problem of the disturbance rejection for multi-agent linear systems to the design of a set of $H_{\infty}$ controllers for a set of independent systems. Liu and Jia (2010 and 2011) derived conditions in terms of linear matrix inequalities (LMIs) to ensure consensus of the multi-agent systems with a prescribed $H_{\infty}$ level. Li, Duan, and Chen (2011) considered the distributed $H_{2}$ and $H_{\infty}$ control problems for linear multi-agent systems. Wen, Duan, Li, and Chen (2012) addressed a finite $\mathrm{L}_{2}$-gain performance index for nonlinear multi-agent systems. Yang and Wang (2013) presented notions of $\mathrm{L}_{\mathrm{p}}$-gain and $\mathrm{L}_{2}$-gain consensus for multi-agent systems in the presence of disturbances.

Most of existing methods for multi-agent control in the presence of disturbances considered the leaderless consensus (or distributed regulation) problem, in which all nodes converge to a common value that cannot generally be controlled. On the other hand, the problem of distributed tracking (or leader-follower consensus problem), which is the problem of interest of this paper, requires that all nodes synchronize to a leader (Hong, $\mathrm{Hu}$, \& Gao, 2006; Li, Wang, \& Chen, 2004; Ren, Moore, \& 
Chen, 2007; Wang, \& Chen, 2002). Moreover, most of these methods derived conditions based on linear matrix inequalities (LMIs) to solve the problem (except for Wen et al. 2012; Yang \& Wang, 2013). A new framework based on differential game theory was developed to achieve consensus of multi-agent systems (Vamvoudakis, Carrillo, \& Hespanha, 2013) and synchronization of multiwheeled mobile robots (Luy, Thanh, \& Tri, 2013). Specifically, Vamvoudakis, Carrillo, and Hespanha (2013) presented a distributed algorithm, but it is limited to double integrator systems. Moreover, the convergence proof of the presented policy iteration algorithm and the $\mathrm{L}_{2}$-bounded synchronization error were not shown.

Over the last decade, multi-agent learning systems have been developed to create agents that learn from experience how to best interact with other agents (Busoniu, Babuska, \& De Schutter, 2008; Chang, 2009; Lakshmanan \& Farias, 2006; Littman, 2001; Vrancx, Verbeeck, \& Nowe, 2008; Wheeler \& Narendra, 1986). A significant part of the research on multi-agent learning concerns reinforcement learning techniques for finite state Markov decision processes. Convergence of each agent to the optimal response using Q-learning was shown on condition that all other agents converge to their optimal response (Littman, 2001). To the best of our knowledge, the multi-agent reinforcement learning for general continuous-time and continuous-state systems in the presence of disturbance has not yet been considered. Moreover, a rigorous proof of the convergence of the multi-agent learning methods to the optimal Nash equilibrium has not yet been provided in the general case.

The main contributions of the paper are as follows. First, a "Bounded $\mathrm{L}_{2}$-Gain Synchronization Problem" is formulated for multi-agent systems in the presence of disturbances in the agent dynamics. Second, the concept of differential game theory is utilized to formulate the distributed $\mathrm{L}_{2}$-Gain control problem into a multi-player zero-sum differential graphical game. Next, it is shown that the solution to this differential graphical game requires the solution to coupled Hamilton-Jacobi-Isaacs (HJI) equations. The Nash solution of the graphical game is investigated and the global synchronization error is shown to be $\mathrm{L}_{2}$-bounded if the graph has a spanning tree. Then, a multi-agent policy iteration algorithm is presented to find the solution to these HJI equations. A rigorous proof of the convergence of the proposed learning algorithm to the optimal Nash equilibrium is presented. This work extends the work of Vamvoudakis, Lewis, and Hudas (2012) to the cases in which the external disturbances cannot be ignored, on one hand, and the work of Vamvoudakis, Carrillo, and Hespanha (2013) to the distributed tracking problem with general linear systems, on the other hand.

This paper is organized as follows. The next section provides mathematical background and the problem formulation for distributed $\mathrm{L}_{2}$-gain control of multi-agent systems. This problem is then formulated into a multiplayer zero-sum differential graphical game in Section 3. The Nash solution to this graphical game is presented in Section 4. Sections 5 and 6 present policy iteration algorithms and their implementation, respectively, for finding the Nash solution to the presented game. Sections 7 and 8 present simulation results and conclusion, respectively.

\section{Preliminaries and Problem Formulation}

In this section, a review of communication graphs is given and the problem of synchronization of multi-agent systems subject to external disturbances is formulated.

\subsection{Mathematical Background}

A directed graph $\mathrm{G}$ consists of a pair $(\mathrm{V}, \mathrm{E})$, where $\mathrm{V}=\left\{\alpha_{1}, \cdots, \alpha_{N}\right\}$ is a finite nonempty set of nodes and $\mathrm{E} \subseteq \mathrm{V} \times \mathrm{V}$ is a set of ordered pairs of nodes, called edges. $E=\left[e_{i j}\right]$ is called the adjacency matrix with $e_{i j}>0$ if $\left(\alpha_{j}, \alpha_{i}\right) \in \mathrm{E}$ and $e_{i j}=0$ otherwise. Note that diagonal elements $e_{i i}=0$. The set of nodes $\alpha_{j}$ with edges incoming to node $\alpha_{i}$ is called the neighbors of node $i$, namely $\mathrm{N}_{i}=\left\{\alpha_{j}:\left(\alpha_{j}, \alpha_{i}\right) \in \mathrm{E}\right\}$. The graph Laplacian matrix is defined as $L=D-E$, which has all row sums equal to zero. $D=\operatorname{diag}\left(d_{i}\right)$ is called the in-degree matrix, where $d_{i}=\sum_{j \in \mathrm{N}_{i}} e_{i j}$ is the weighted in-degree of node $i$.

Definition 1. (Lewis et al., 2014) A (directed) tree is a connected digraph where every node except one, called the root, has in-degree equal to one. A graph is said to have a spanning tree if a subset of the edges forms a directed tree.

Throughout the paper, $\bar{\sigma}(A)$ and $\sigma(A)$ are denoted as the maximum and minimum singular values of the matrix $A$, respectively.

\subsection{Problem Formulation}

Consider the communication graph $\mathrm{G}=(\mathrm{V}, \mathrm{E})$ having $N$ agents, each with dynamics given by

$$
\dot{x}_{i}=A x_{i}+B_{i} u_{i}+D_{i} v_{i}
$$

where $x_{i}(t) \in R^{n}, u_{i}(t) \in R^{m_{i}}$ and $v_{i}(t) \in R^{q_{i}}$ are the state, control input and external disturbance of node $i$, respectively. The state of the control or leader node is $x_{0}(t) \in R^{n}$ and it is assumed to satisfy the dynamics

$$
\dot{x}_{0}=A x_{0}
$$

Standard Synchronization Problem. Design $u_{i}(t)$ in (1) so that $\left\|x_{i}(t)-x_{0}(t)\right\| \rightarrow 0, \forall i$ when $v_{i}(t)=0$.

For each node $i$, the local neighborhood tracking error $\delta_{i} \in R^{n}$ is defined as (Khoo, Xie, \& Man, 2009)

$$
\delta_{i}=\sum_{j \in \mathrm{N}_{i}} e_{i j}\left(x_{i}-x_{j}\right)+g_{i}\left(x_{i}-x_{0}\right)
$$

where $g_{i} \geq 0$ is called the pinning gain and $g_{i}>0$ for at least one root node $i$ (Li, Wang, \& Chen, 2004). by

The overall tracking error vector for all nodes is given

$$
\delta=\left((L+G) \otimes I_{n}\right)\left(x-\underline{x}_{0}\right)=\left((L+G) \otimes I_{n}\right) \zeta
$$

where $x=\left[\begin{array}{llll}x_{1}^{T} & x_{2}^{T} & \cdots & x_{N}^{T}\end{array}\right]^{T}, \delta=\left[\begin{array}{llll}\delta_{1}^{T} & \delta_{2}^{T} & \cdots & \delta_{N}^{T}\end{array}\right]^{T}$ are global node state vector and the global tracking error vector, and $\underline{x}_{0}=\underline{I} x_{0} \in R^{n N}$, with $\underline{I}=\mathbf{1}_{N} \otimes I_{n} \in R^{n N \times n}, I_{n}$ the $n \times n$ identity matrix and $\mathbf{1}_{N}$ the $N$-vector of ones. The 
Kronecker product is denoted by $\otimes$. The pinning gain matrix $G \in R^{N \times N}$ is a diagonal matrix with diagonal entries equal to the pinning gains $g_{i}$. The (global) synchronization error is

$$
\zeta=\left(x-\underline{x}_{0}\right) \in R^{n N}
$$

The following Lemma shows that the small local neighborhood synchronization error implies small global synchronization error.

Lemma 1. (Khoo, Xie, \& Man, 2009) Let $(L+G)$ be nonsingular. Then the synchronization error is bounded by

$$
\|\zeta\| \leq\|\delta\| / \underline{\sigma}(L+G)
$$

Remark 1. The matrix $(L+G)$ is non-singular if the graph has a spanning tree and $g_{i} \neq 0$ for a root node $i$ (Khoo, Xie, \& Man, 2009)

The local neighborhood tracking error dynamics for node $i$ is defined as

$$
\dot{\delta}_{i}=\sum_{j \in \mathrm{N}_{i}} e_{i j}\left(\dot{x}_{i}-\dot{x}_{j}\right)+g_{i}\left(\dot{x}_{i}-\dot{x}_{0}\right)
$$

which by using (1) and (2) becomes

$$
\begin{aligned}
\dot{\delta}_{i}= & A \delta_{i}+\left(d_{i}+g_{i}\right) B_{i} u_{i}-\sum_{j \in \mathrm{N}_{i}} e_{i j} B_{j} u_{j}+\left(d_{i}+g_{i}\right) D_{i} v_{i} \\
& -\sum_{j \in \mathrm{N}_{i}} e_{i j} D_{j} v_{j}
\end{aligned}
$$

with $\delta_{i} \in R^{n}, u_{i} \in R^{m_{i}}, v_{i} \in R^{q_{i}}, \forall i$. This is a dynamical system with multiple control inputs and disturbance inputs from node $i$ and all of her neighbors.

The objective here is to design a distributed control protocol to make the tracking synchronization error (3) $\mathrm{L}_{2}$-bounded when $v_{i}(t) \neq 0$.

Bounded $\mathbf{L}_{2}$-Gain Synchronization Problem. Consider the systems (8) with measured outputs $y_{i}=H_{i i} \delta_{i}$ (Note that $H_{i i}$ must be left invertible and hence $\delta_{i}$ can be directly measured), disturbances $\omega_{i}(t)=\left[\begin{array}{lll}v_{i}^{T}(t) & v_{-i}^{T}(t)\end{array}\right]^{T}$ and performance outputs $z_{i}(t)=\left[\begin{array}{llll}\delta_{i}^{T}(t) & u_{i}^{T}(t) & u_{-i}{ }^{-i}(t)\end{array}\right]^{T}$ with $v_{-i}(t)=\left\{v_{j}: j \in \mathrm{N}_{i}\right\}$ and $u_{-i}(t)=\left\{u_{j}: j \in \mathrm{N}_{i}\right\}$. It is desired to select the control $u_{i}(t)$ to solve the standard synchronization problem when $v_{i}(t)=0$, and also to satisfy the following bounded $\mathrm{L}_{2}$-gain condition for a given $\gamma>0$ for all agents when $v_{i}(t) \neq 0$,

$$
\begin{aligned}
\int_{0}^{T}\left\|z_{i}(t)\right\|^{2} d t & =\int_{0}^{T}\left(\delta_{i}^{T} Q_{i i} \delta_{i}+u_{i}^{T} R_{i i} u_{i}+\sum_{j \in \mathrm{N}_{i}} u_{j}^{T} R_{i j} u_{j}\right) d t \\
& \leq \gamma^{2} \int_{0}^{T}\left\|\omega_{i}(t)\right\|^{2} d t+\beta\left(\delta_{i}(0)\right) \\
& =\gamma^{2} \int_{0}^{T}\left(v_{i}^{T} T_{i i} v_{i}+\sum_{j \in \mathrm{N}_{i}} v_{j}^{T} T_{i j} v_{j}\right) d t+\beta\left(\delta_{i}(0)\right)
\end{aligned}
$$

for some bounded function $\beta$ such that $\beta(0)=0$ (Aliyu, 2011), with $Q_{i i}>0, R_{i i}>0, R_{i j} \geq 0, T_{i i}>0, T_{i j} \geq 0$. Let $\gamma^{*}$ be the minimum value of $\gamma$ for which the disturbance attenuation condition (9) is satisfied.

\section{Multi-Player Zero-Sum Differential Graphical Game}

Define the following local performance index for each agent.

$$
\begin{aligned}
& J_{i}\left(\delta_{i}(0), u_{i}, u_{-i}, v_{i}, v_{-i}\right)=\frac{1}{2} \int_{0}^{\infty}\left(\delta_{i}^{T} Q_{i i} \delta_{i}+u_{i}^{T} R_{i i} u_{i}\right. \\
& \left.+\sum_{j \in \mathrm{N}_{i}} u_{j}^{T} R_{i j} u_{j}-\gamma^{2} v_{i}^{T} T_{i i} v_{i}-\gamma^{2} \sum_{j \in \mathrm{N}_{i}} v_{j}^{T} T_{i j} v_{j}\right) d t
\end{aligned}
$$

\subsection{Zero-Sum Differential Graphical Games}

It will be shown in section 4 that the solvability of the bounded $\mathrm{L}_{2}$-gain synchronization problem is equivalent to the solvability of the following multi-player zero-sum differential game

$$
V_{i}\left(\delta_{i}(0)\right)=\min _{u_{i}} \max _{v_{i}} J_{i}\left(\delta_{i}(0), u_{i}, u_{-i}, v_{i}, v_{-i}\right)
$$

where $J_{i}$ is defined in (10). The control player seeks to minimize the value and the disturbance player to maximize it. This game has a unique solution if a game theoretic saddle point $\left(u_{i}^{*}, v_{i}^{*}\right)$ exists. That is, if (Basar \& Bernard, 2008; Lewis, Vrabie, \& Syrmos, 2012)

$$
\begin{aligned}
V_{i}^{*}\left(\delta_{i}(0)\right) & =\min _{u_{i}} \max _{v_{i}} J_{i}\left(\delta_{i}(0), u_{i}, u_{-i}^{*}, v_{i}, v_{-i}^{*}\right) \\
& =\max _{v_{i}} \min _{u_{i}} J_{i}\left(\delta_{i}(0), u_{i}, u_{-i}^{*}, v_{i}, v_{-i}^{*}\right)
\end{aligned}
$$

The associated value $V_{i}^{*}$ is called the value of the game. This is equivalent to the Nash equilibrium condition

$$
J_{i}\left(u_{i}^{*}, u_{-i}^{*}, v_{i}, v_{-i}^{*}\right) \leq J_{i}\left(u_{i}^{*}, u_{-i}^{*}, v_{i}^{*}, v_{-i}^{*}\right) \leq J_{i}\left(u_{i}, u_{-i}^{*}, v_{i}^{*}, v_{-i}^{*}\right)
$$

holding for all policies $u_{i}, v_{i}$. Interpreting the control inputs $u_{i}, \forall i$, and the disturbance inputs $v_{i}, \forall i$ as policies in terms of $\delta_{i}$, the value function for node $i$ is

$$
\begin{array}{r}
V_{i}\left(\delta_{i}(t)\right)=\frac{1}{2} \int_{t}^{\infty}\left(\delta_{i}^{T} Q_{i i} \delta_{i}+u_{i}^{T} R_{i i} u_{i}+\sum_{j \in \mathrm{N}_{i}} u_{j}^{T} R_{i j} u_{j}\right. \\
\left.-\gamma^{2} v_{i}^{T} T_{i i} v_{i}-\gamma^{2} \sum_{j \in \mathrm{N}_{i}} v_{j}^{T} T_{i j} v_{j}\right) d t
\end{array}
$$

Using Leibniz's formula, a differential equivalent to (14) is given by the Bellman equation as

$$
\begin{aligned}
& H_{i}\left(\delta_{i}, \frac{\partial V_{i}}{\partial \delta_{i}}, u_{i}, u_{-i}, v_{i}, v_{-i}\right) \equiv \frac{\partial V_{i}^{T}}{\partial \delta_{i}}\left(A \delta_{i}+\left(d_{i}+g_{i}\right) B_{i} u_{i}\right. \\
& \left.-\sum_{j \in \mathrm{N}_{i}} e_{i j} B_{j} u_{j}+\left(d_{i}+g_{i}\right) D_{i} v_{i}-\sum_{j \in \mathrm{N}_{i}} e_{i j} D_{j} v_{j}\right)+\frac{1}{2} \delta_{i}^{T} Q_{i i} \delta_{i} \\
& +\frac{1}{2} u_{i}^{T} R_{i i} u_{i}+\frac{1}{2} \sum_{j \in \mathrm{N}_{i}} u_{j}^{T} R_{i j} u_{j}-\frac{1}{2} \gamma^{2} v_{i}^{T} T_{i i} v_{i}-\frac{1}{2} \gamma^{2} \sum_{j \in \mathrm{N}_{i}} v_{j}^{T} T_{i j} v_{j}=0
\end{aligned}
$$

with the boundary condition $V_{i}(0)=0$. The stationarity conditions give (Lewis, Vrabie, \& Syrmos, 2012)

$$
\begin{aligned}
& 0=\frac{\partial H_{i}}{\partial u_{i}} \Rightarrow u_{i}=-\left(d_{i}+g_{i}\right) R_{i i}^{-1} B_{i}^{T} \frac{\partial V_{i}}{\partial \delta_{i}} \\
& 0=\frac{\partial H_{i}}{\partial v_{i}} \Rightarrow v_{i}=\frac{1}{\gamma^{2}}\left(d_{i}+g_{i}\right) T_{i i}^{-1} D_{i}^{T} \frac{\partial V_{i}}{\partial \delta_{i}}
\end{aligned}
$$


Remark 2. The game defined by (10), (11) is in fact a zero-sum game with respect to $u_{i}$ and $v_{i}$ for each agent $i$, with outcome coupled through the neighboring relations expressed by the sets $\mathrm{N}_{i}$.

\subsection{Coupled Hamilton-Jacobi-Isaacs Equations}

Substituting control policies (16) and disturbance policies (17) into (15) yields the coupled HJI equations

$$
\begin{aligned}
& \frac{\partial V_{i}^{T}}{\partial \delta_{i}} A_{i}^{c}+\frac{1}{2} \delta_{i}^{T} Q_{i i} \delta_{i}+\frac{1}{2}\left(d_{i}+g_{i}\right)^{2} \frac{\partial V_{i}^{T}}{\partial \delta_{i}} B_{i} R_{i i}^{-1} B_{i}^{T} \frac{\partial V_{i}}{\partial \delta_{i}} \\
& +\frac{1}{2} \sum_{j \in \mathrm{N}_{i}}\left(d_{j}+g_{j}\right)^{2} \frac{\partial V_{j}^{T}}{\partial \delta_{j}} B_{j} R_{j j}^{-1} R_{i j} R_{j j}^{-1} B_{j}^{T} \frac{\partial V_{j}}{\partial \delta_{j}} \\
& -\frac{1}{2 \gamma^{2}}\left(d_{i}+g_{i}\right)^{2} \frac{\partial V_{i}^{T}}{\partial \delta_{i}} D_{i} T_{i i}^{-1} D_{i}^{T} \frac{\partial V_{i}}{\partial \delta_{i}} \\
& -\frac{1}{2 \gamma^{2}} \sum_{j \in \mathrm{N}_{i}}\left(d_{j}+g_{j}\right)^{2} \frac{\partial V_{j}^{T}}{\partial \delta_{j}} D_{j} T_{j j}^{-1} T_{i j} T_{j j}^{-1} D_{j}^{T} \frac{\partial V_{j}}{\partial \delta_{j}}=0
\end{aligned}
$$

with boundary condition $V_{i}(0)=0$, where

$$
\begin{aligned}
A_{i}^{c}= & A \delta_{i}-\left(d_{i}+g_{i}\right)^{2} B_{i} R_{i i}^{-1} B_{i}{ }^{T} \frac{\partial V_{i}}{\partial \delta_{i}}+\sum_{j \in \mathrm{N}_{i}} e_{i j}\left(d_{j}+g_{j}\right) \times \\
& B_{j} R_{j j}^{-1} B_{j}{ }^{T} \frac{\partial V_{j}}{\partial \delta_{j}}+\frac{1}{\gamma^{2}}\left(d_{i}+g_{i}\right)^{2} D_{i} T_{i i}^{-1} D_{i}{ }^{T} \frac{\partial V_{i}}{\partial \delta_{i}} \\
& -\frac{1}{\gamma^{2}} \sum_{j \in \mathrm{N}_{i}} e_{i j}\left(d_{j}+g_{j}\right) D_{j} T_{j j}^{-1} D_{j}{ }^{T} \frac{\partial V_{j}}{\partial \delta_{j}}
\end{aligned}
$$

is the closed-loop system corresponding to agent $i$. For a given solution $V_{i}$, define $u_{i}^{*}=u_{i}\left(V_{i}\right), u_{-i}^{*}=u_{-i}\left(V_{i}\right)$, $v_{i}^{*}=v_{i}\left(V_{i}\right)$ and $v_{-i}^{*}=v_{-i}\left(V_{i}\right)$ in terms of $V_{i}$, then (18) can be written as

$$
H_{i}\left(\delta_{i}, \frac{\partial V_{i}}{\partial \delta_{i}}, u_{i}^{*}, u_{-i}^{*}, v_{i}^{*}, v_{-i}^{*}\right)=0, V_{i}(0)=0
$$

Lemma 2. Assume that the neighboring agents' policies are already optimal. Then, for every $u_{i}, v_{i}$, the following holds

$$
\begin{aligned}
& H_{i}\left(\delta_{i}, \frac{\partial V_{i}}{\partial \delta_{i}}, u_{i}, u_{-i}^{*}, v_{i}, v_{-i}^{*}\right)=\frac{1}{2}\left(u_{i}-u_{i}^{*}\right)^{T} R_{i i}\left(u_{i}-u_{i}^{*}\right) \\
&-\frac{1}{2} \gamma^{2}\left(v_{i}-v_{i}^{*}\right)^{T} T_{i i}\left(v_{i}-v_{i}^{*}\right)
\end{aligned}
$$

Proof. Completing the squares in (15) gives (20).

\section{4. $L_{2}$-Bounded Synchronization Error and Nash Solution to the Graphical Games}

\section{1. $L_{2}$-Bounded Synchronization Error}

In this subsection, we want to find the control inputs $u_{i}(t)$ such that, for all disturbances $v_{i}(t) \in \mathrm{L}_{2}[0, \infty)$ the condition (9) holds for a prescribed $\gamma>0$. The following result provides a solution to the bounded $\mathrm{L}_{2}$-gain problem in terms of a solution to the HJI equation (18).
Theorem 1. Solution to Bounded $L_{2}$-gain Problem. Let $\gamma \geq \gamma^{*}$. Suppose $V_{i}^{*}>0, i \in N$ is a smooth positive definite solution to the HJI equation (18). Assume that the neighboring agents' policies are already optimal. Then, the equilibrium point of the closed-loop system

$$
\dot{\delta}_{i}=A \delta_{i}+\left(d_{i}+g_{i}\right) B_{i} u_{i}^{*}-\sum_{j \in \mathrm{N}_{i}} e_{i j} B_{j} u_{j}^{*}
$$

is asymptotically stable with control inputs $u_{i}^{*}=u_{i}\left(V_{i}^{*}\right)$, given by (16) in terms of $V_{i}^{*}$. Moreover, for these choices of control inputs, the bounded $\mathrm{L}_{2}$-gain condition (9) holds for all disturbances $v_{i}(t) \in \mathrm{L}_{2}[0, \infty)$, where $Q_{i i}=H_{i i}^{T} H_{i i}$.

Proof. Suppose now that $V_{j}^{*}$ satisfies the coupled HJI equation (18) and set $u_{-i}=u_{-i}^{*}, v_{-i}=v_{-i}^{*}$. Then, using (20) in the Hamiltonian function (15) in terms of $V_{i}^{*}$, one has

$$
\begin{aligned}
& H_{i}\left(\delta_{i}, \frac{\partial V_{i}^{*}}{\partial \delta_{i}}, u_{i}, u_{-i}, v_{i}, v_{-i}\right)=\frac{d V_{i}^{*}}{d t}+\frac{1}{2} \delta_{i}^{T} Q_{i i} \delta_{i}+\frac{1}{2} u_{i}^{T} R_{i i} u_{i} \\
& +\frac{1}{2} \sum_{j \in \mathrm{N}_{i}} u_{j}^{T} R_{i j} u_{j}-\frac{1}{2} \gamma^{2} v_{i}^{T} T_{i i} v_{i}-\frac{1}{2} \gamma^{2} \sum_{j \in \mathrm{N}_{i}} v_{j}^{T} T_{i j} v_{j} \\
& =\frac{1}{2}\left(u_{i}-u_{i}^{*}\right)^{T} R_{i i}\left(u_{i}-u_{i}^{*}\right)-\frac{1}{2} \gamma^{2}\left(v_{i}-v_{i}^{*}\right)^{T} T_{i i}\left(v_{i}-v_{i}^{*}\right)
\end{aligned}
$$

Selecting $u_{i}=u_{i}^{*}$ yields

$$
\begin{aligned}
& \frac{d V_{i}^{*}}{d t}+\frac{1}{2} \delta_{i}^{T} Q_{i i} \delta_{i}+\frac{1}{2} u_{i}^{T} R_{i i} u_{i}+\frac{1}{2} \sum_{j \in \mathrm{N}_{i}} u_{j}^{T} R_{i j} u_{j} \\
& -\frac{1}{2} \gamma^{2} v_{i}^{T} T_{i i} v_{i}-\frac{1}{2} \gamma^{2} \sum_{j \in \mathrm{N}_{i}} v_{j}^{T} T_{i j} v_{j} \leq 0
\end{aligned}
$$

for all $v_{i}, v_{j}$. To show asymptotic stability of the closedloop system (21), set $v_{i}=v_{j}=0$ in the above equation. Then, one has

$$
\frac{d V_{i}^{*}}{d t} \leq-\frac{1}{2}\left(\delta_{i}^{T} Q_{i i} \delta_{i}+u_{i}^{T} R_{i i} u_{i}+\sum_{j \in \mathrm{N}_{i}} u_{j}^{T} R_{i j} u_{j}\right) \leq-\frac{1}{2} \delta_{i}^{T} Q_{i i} \delta_{i}
$$

Therefore, the equilibrium point of the system is asymptotically stable using the Lyapunov Theorem. To show that the condition (9) is satisfied, integrating (22) yields,

$$
\begin{aligned}
& V_{i}^{*}\left(\delta_{i}(t)\right)-V_{i}^{*}\left(\delta_{i}(0)\right)+\frac{1}{2} \int_{0}^{T}\left(\delta_{i}^{T} Q_{i i} \delta_{i}+u_{i}^{T} R_{i i} u_{i}\right. \\
& \left.+\sum_{j \in \mathrm{N}_{i}} u_{j}^{T} R_{i j} u_{j}-\gamma^{2} v_{i}^{T} T_{i i} v_{i}-\gamma^{2} \sum_{j \in \mathrm{N}_{i}} v_{j}^{T} T_{i j} v_{j}\right) d t \leq 0
\end{aligned}
$$

Noting that $V_{i}^{*}\left(\delta_{i}(t)\right)>0$, one has

$$
\begin{aligned}
& \int_{0}^{T}\left(\delta_{i}^{T} Q_{i i} \delta_{i}+u_{i}^{T} R_{i i} u_{i}+\sum_{j \in \mathrm{N}_{i}} u_{j}^{T} R_{i j} u_{j}\right) d t \\
& \leq \gamma^{2} \int_{0}^{T}\left(v_{i}^{T} T_{i i} v_{i}+\sum_{j \in \mathrm{N}_{i}} v_{j}^{T} T_{i j} v_{j}\right) d t+V_{i}^{*}\left(\delta_{i}(0)\right)
\end{aligned}
$$

Therefore, the $L_{2}$ gain is bounded by $\gamma$ and (9) is satisfied.

Corollary 1. Assume that the assumptions made in the statement of Theorem 1 are satisfied. Then, the global synchronization error $\zeta$ is $\mathrm{L}_{2}$-bounded, if the graph has a spanning tree.

Proof. By Theorem 1 and Lemma 1, one can conclude that the global synchronization error is $\mathrm{L}_{2}$-bounded, if the graph has a spanning tree. 


\subsection{Nash Solution to the Graphical Game}

The following result shows that under certain conditions the HJI solution satisfies the Nash condition (13) and so solves the zero-sum game.

Theorem 2. Solution to multi-player zero-sum game. Let $\gamma \geq \gamma^{*}$. Assume that the game (11) has a finite value and the neighboring agents' policies are already optimal. Suppose $V_{i}^{*}>0, i \in N$ is a smooth positive definite solution to the HJI equation (18) such that the equilibrium point of the closed-loop system

$$
\begin{aligned}
\dot{\delta}_{i}= & A \delta_{i}+\left(d_{i}+g_{i}\right) B_{i} u_{i}^{*}-\sum_{j \in \mathrm{N}_{i}} e_{i j} B_{j} u_{j}^{*} \\
& +\left(d_{i}+g_{i}\right) D_{i} v_{i}^{*}-\sum_{j \in \mathrm{N}_{i}} e_{i j} D_{j} v_{j}^{*}
\end{aligned}
$$

is asymptotically stable. Then, the Nash condition (13) is satisfied for control policy $u_{i}^{*}=u_{i}\left(V_{i}^{*}\right)$ and disturbance policy $v_{i}^{*}=v_{i}\left(V_{i}^{*}\right)$ as (16) and (17), given in terms of $V_{i}^{*}$. Moreover, the value of the game is given by the HJI solution $V_{i}^{*}\left(\delta_{i}(0)\right)$.

Proof. For any smooth functions $V_{i}\left(\delta_{i}\right), i \in N$, one can write (10) as

$$
\begin{aligned}
& J_{i}\left(\delta_{i}(0), u_{i}, u_{-i}, v_{i}, v_{-i}\right) \\
= & \frac{1}{2} \int_{0}^{\infty}\left(\delta_{i}^{T} Q_{i i} \delta_{i}+u_{i}^{T} R_{i i} u_{i}+\sum_{j \in \mathrm{N}_{i}} u_{j}^{T} R_{i j} u_{j}-\gamma^{2} v_{i}^{T} T_{i i} v_{i}\right. \\
& \left.-\gamma^{2} \sum_{j \in \mathrm{N}_{i}} v_{j}^{T} T_{i j} v_{j}\right) d t+V_{i}\left(\delta_{i}(0)\right)-V_{i}\left(\delta_{i}(\infty)\right)+\int_{0}^{\infty} \frac{\partial V_{i}^{T}}{\partial \delta_{i}} \\
& \left(A \delta_{i}+\left(d_{i}+g_{i}\right) B_{i} u_{i}-\sum_{j \in \mathrm{N}_{i}} e_{i j} B_{j} u_{j}+\left(d_{i}+g_{i}\right) D_{i} v_{i}\right. \\
& \left.-\sum_{j \in \mathrm{N}_{i}} e_{i j} D_{j} v_{j}\right) d t
\end{aligned}
$$

Now let $V_{i}^{*}$ satisfies (18) and $u_{i}^{*}, u_{-i}^{*}, v_{i}^{*}, v_{-i}^{*}$ be the optimal policies given by (16) and (17). Completing the squares yields as

$$
\begin{aligned}
& J_{i}\left(\delta_{i}(0), u_{i}, u_{-i}, v_{i}, v_{-i}\right) \\
= & V_{i}^{*}\left(\delta_{i}(0)\right)-V_{i}^{*}\left(\delta_{i}(\infty)\right)+\int_{0}^{\infty}\left(\frac{1}{2} \sum_{j \in \mathrm{N}_{i}}\left(u_{j}-u_{j}^{*}\right)^{T} R_{i j}\left(u_{j}-u_{j}^{*}\right)\right. \\
+ & \frac{1}{2}\left(u_{i}-u_{i}^{*}\right)^{T} R_{i i}\left(u_{i}-u_{i}^{*}\right)-\frac{\partial V_{i}^{T}}{\partial \delta_{i}} \sum_{j \in \mathrm{N}_{i}} e_{i j} B_{j}\left(u_{j}-u_{j}^{*}\right) \\
+ & \sum_{j \in \mathrm{N}_{i}} u_{j}^{* T} R_{i j}\left(u_{j}-u_{j}^{*}\right)-\frac{1}{2} \gamma^{2} \sum_{j \in \mathrm{N}_{i}}\left(v_{j}-v_{j}^{*}\right)^{T} T_{i j}\left(v_{j}-v_{j}^{*}\right) \\
- & \frac{1}{2} \gamma^{2}\left(v_{i}-v_{i}^{*}\right)^{T} T_{i i}\left(v_{i}-v_{i}^{*}\right)-\frac{\partial V_{i}^{T}}{\partial \delta_{i}} \sum_{j \in \mathrm{N}_{i}} e_{i j} D_{j}\left(v_{j}-v_{j}^{*}\right) \\
- & \left.\gamma^{2} \sum_{j \in \mathrm{N}_{i}} v_{j}^{* T} T_{i j}\left(v_{j}-v_{j}^{*}\right)\right) d t
\end{aligned}
$$

Since the equilibrium point of the closed-loop system (23) is asymptotically stable, one has $V_{i}^{*}\left(\delta_{i}(\infty)\right)=0$.
Assuming $u_{j}=u_{j}^{*}, v_{j}=v_{j}^{*}, \forall j \in \mathrm{N}_{i}$, then we obtain

$$
\begin{aligned}
& J_{i}\left(\delta_{i}(0), u_{i}, u_{-i}^{*}, v_{i}, v_{-i}^{*}\right)=V_{i}^{*}\left(\delta_{i}(0)\right)+\int_{0}^{\infty}\left(\frac{1}{2}\left(u_{i}-u_{i}^{*}\right)^{T}\right. \\
& \left.R_{i i}\left(u_{i}-u_{i}^{*}\right)-\frac{1}{2} \gamma^{2}\left(v_{i}-v_{i}^{*}\right)^{T} T_{i i}\left(v_{i}-v_{i}^{*}\right)\right) d t
\end{aligned}
$$

Due to its quadratic form, (26) shows the Nash condition (13) is satisfied. In fact, if the strategy of the disturbance input (the control input) remains unchanged, the control input (the disturbance input) does not gain anything based on (26). Moreover, setting $u_{i}=u_{i}^{*}, v_{i}=v_{i}^{*}$ in (26) leads to

$$
J_{i}^{*}\left(\delta_{i}(0), u_{i}^{*}, u_{-i}^{*}, v_{i}^{*}, v_{-i}^{*}\right)=V_{i}^{*}\left(\delta_{i}(0)\right)
$$

which gives the optimal game value. This completes the proof.

\section{Multi-agent Policy Iteration Algorithm to solve HJI equations}

\subsection{Best response}

Definition 2. Given fixed neighbor control policies $u_{-i}$ and disturbance policies $v_{-i}$, assume that there exist stabilizing control policy $u_{i}$ and disturbance policy $v_{i}$. Then, agent $i$ 's best control and disturbance response to fixed policies $u_{-i}$ and $v_{-i}$ of her neighbors are the pair of policies $u_{i}^{*}$ and $v_{i}^{*}$ such that

$$
J_{i}\left(u_{i}^{*}, u_{-i}, v_{i}, v_{-i}\right) \leq J_{i}\left(u_{i}^{*}, u_{-i}, v_{i}^{*}, v_{-i}\right) \leq J_{i}\left(u_{i}, u_{-i}, v_{i}^{*}, v_{-i}\right)
$$

Define the best response HJI equation with control $u_{i}=u_{i}^{*}$ given by (16) and disturbances $v_{i}=v_{i}^{*}$ given by (17) and arbitrary policies $u_{-i}, v_{-i}$ as

$$
\begin{aligned}
& \frac{\partial V_{i}^{T}}{\partial \delta_{i}} A_{i}^{c}+\frac{1}{2} \delta_{i}^{T} Q_{i i} \delta_{i}+\frac{1}{2}\left(d_{i}+g_{i}\right)^{2} \frac{\partial V_{i}^{T}}{\partial \delta_{i}} B_{i} R_{i i}^{-1} B_{i}^{T} \frac{\partial V_{i}}{\partial \delta_{i}} \\
& +\frac{1}{2} \sum_{j \in \mathrm{N}_{i}} u_{j}^{T} R_{i j} u_{j}-\frac{1}{2 \gamma^{2}}\left(d_{i}+g_{i}\right)^{2} \frac{\partial V_{i}^{T}}{\partial \delta_{i}} D_{i} T_{i i}^{-1} D_{i}^{T} \frac{\partial V_{i}}{\partial \delta_{i}} \\
& -\frac{1}{2} \gamma^{2} \sum_{j \in \mathrm{N}_{i}} v_{j}^{T} T_{i j} v_{j}=0
\end{aligned}
$$

with boundary condition $V_{i}(0)=0$, where $A_{i}^{c}, \forall i$ is the closed-loop system.

\subsection{Policy iteration to solve the HJI equations}

In this subsection, a policy iteration (PI) algorithm is presented to solve the best response HJI equations (29) and the HJI equations (18). The PI technique has two loops, i.e. an inner loop (policy evaluation) and an outer loop (policy improvement). In the inner loop, the control policies are fixed and the disturbances are updated using (17) by solving the Bellman equation (15). In the outer loop, improved control policies are found using (16). This gives the following PI algorithm.

Algorithm 1: Multi-agent Learning Policy Iteration for Solving HJI equations

Let $u_{i}^{0}, \forall i=1, \ldots, N$ be any stabilizing initial policy. 
For $k=0,1, \ldots$, given $u_{i}^{k}, \forall i=1, \ldots, N$.

repeat at each step $k$

for $l=0,1, \ldots$, set $v_{i}^{0}=0$, (Policy evaluation) repeat at each step $l$

solve for $V_{i}^{(k, l)}\left(\delta_{i}\right)$ using the zero-sum Bellman equation

$$
\begin{aligned}
& \left(\frac{\partial V_{i}^{(k, l)}}{\partial \delta_{i}}\right)^{T}\left(A \delta_{i}+\left(d_{i}+g_{i}\right) B_{i} u_{i}^{k}-\sum_{j \in \mathrm{N}_{i}} e_{i j} B_{j} u_{j}\right. \\
& \left.+\left(d_{i}+g_{i}\right) D_{i} v_{i}^{l}-\sum_{j \in \mathrm{N}_{i}} e_{i j} D_{j} v_{j}\right)+\frac{1}{2} \delta_{i}^{T} Q_{i i} \delta_{i} \\
& +\frac{1}{2} u_{i}^{k T} R_{i i} u_{i}^{k}+\frac{1}{2} \sum_{j \in \mathrm{N}_{i}} u_{j}^{T} R_{i j} u_{j}-\frac{1}{2} \gamma^{2} v_{i}^{l T} T_{i i} v_{i}^{l} \\
& -\frac{1}{2} \gamma^{2} \sum_{j \in \mathrm{N}_{i}} v_{j}^{T} T_{i j} v_{j}=0 \quad \forall i=1, \ldots, N
\end{aligned}
$$

update the disturbances $v_{i}^{l+1}$ according to

$$
v_{i}^{l+1}=\frac{1}{\gamma^{2}}\left(d_{i}+g_{i}\right) T_{i i}^{-1} D_{i}^{T} \frac{\partial V_{i}^{(k, l)}}{\partial \delta_{i}} \forall i=1, \ldots, N
$$

until convergence, set $V_{i}^{(k+1, l)}=V_{i}^{(k, l)}$ end for

update the control policy (Policy improvement) using

$$
u_{i}^{k+1}=-\left(d_{i}+g_{i}\right) R_{i i}^{-1} B_{i}^{T} \frac{\partial V_{i}^{(k, l)}}{\partial \delta_{i}} \forall i=1, \ldots, N
$$

until convergence

\section{end for}

The following two theorems prove the convergence of the policy iteration Algorithm 1 for the best response HJI equation (29) and the coupled HJI equation (18), respectively. In Theorem 3 , only agent $i$ updates her policy and in Theorem 4, all the agents update their policies simultaneously.

Theorem 3. Assume that the policies in the neighborhood are fixed and that there exist a stabilizing control policy and disturbance policy with $\gamma \geq \gamma^{*}$. Assume that agent $i$ performs Algorithm 1. Then the algorithm converges to the best response $u_{i}$ and $v_{i}$ to policies $u_{-i}$ and $v_{-i}$ of the neighbors, and the value converges to the optimal game value $V_{i}^{(k, l)} \rightarrow V_{i}^{*}$, which $V_{i}^{*}$ is the solution to the best response HJI equation (29).

Proof. Firstly, we prove the convergence of the inner-loop By integrating $\dot{V}_{i}^{(k, l)}-\dot{V}_{i}^{(k, l+1)}$ along the solution of the error system

$$
\begin{aligned}
\dot{\delta}_{i}= & A \delta_{i}+\left(d_{i}+g_{i}\right) B_{i} u_{i}^{k}-\sum_{j \in \mathrm{N}_{i}} e_{i j} B_{j} u_{j} \\
& +\left(d_{i}+g_{i}\right) D_{i} v_{i}^{l+1}-\sum_{j \in \mathrm{N}_{i}} e_{i j} D_{j} v_{j}
\end{aligned}
$$

and considering that only agent $i$ updates her policy, yields

$$
\begin{aligned}
& V_{i}^{(k, l)}-V_{i}^{(k, l+1)}=\int_{t}^{\infty}\left(( \frac { \partial V _ { i } ^ { ( k , l + 1 ) } } { \partial \delta _ { i } } ) ^ { T } \left(A \delta_{i}+\left(d_{i}+g_{i}\right) B_{i} u_{i}^{k}\right.\right. \\
& \left.-\sum_{j \in \mathrm{N}_{i}} e_{i j} B_{j} u_{j}+\left(d_{i}+g_{i}\right) D_{i} v_{i}^{l+1}-\sum_{j \in \mathrm{N}_{i}} e_{i j} D_{j} v_{j}\right) \\
& -\left(\frac{\partial V_{i}^{(k, l)}}{\partial \delta_{i}}\right)^{T}\left(A \delta_{i}+\left(d_{i}+g_{i}\right) B_{i} u_{i}^{k}-\sum_{j \in \mathrm{N}_{i}} e_{i j} B_{j} u_{j}\right. \\
& \left.\left.+\left(d_{i}+g_{i}\right) D_{i} v_{i}^{l+1}-\sum_{j \in \mathrm{N}_{i}} e_{i j} D_{j} v_{j}\right)\right) d t
\end{aligned}
$$

Using (30) for the first and second terms of the right-hand side the above equation one has

$$
\begin{aligned}
& V_{i}^{(k, l)}-V_{i}^{(k, l+1)}=\int_{t}^{\infty}\left(\frac{1}{2} \gamma^{2} v_{i}^{(l+1) T} T_{i i} v_{i}^{(l+1)}-\frac{1}{2} \gamma^{2} v_{i}^{l T} T_{i i} v_{i}^{l}\right. \\
& \left.+\left(\frac{\partial V_{i}^{(k, l)}}{\partial \delta_{i}}\right)^{T}\left(d_{i}+g_{i}\right) D_{i} v_{i}^{l}-\left(\frac{\partial V_{i}^{(k, l)}}{\partial \delta_{i}}\right)^{T}\left(d_{i}+g_{i}\right) D_{i} v_{i}^{l+1}\right) d t
\end{aligned}
$$

Then, substituting (31) into (35) and doing some manipulations gives

$$
V_{i}^{(k, l)}-V_{i}^{(k, l+1)}=-\int_{t}^{\infty}\left(\frac{1}{2} \gamma^{2}\left(v_{i}^{(l+1)}-v_{i}^{l}\right)^{T} T_{i i}\left(v_{i}^{(l+1)}-v_{i}^{l}\right)\right) d t \leq 0
$$

Therefore, one has $V_{i}^{(k, l)} \leq V_{i}^{(k, l+1)}$. Defining the sequence $a_{l}=V_{i}^{(k, l)}$, the inequality $V_{i}^{(k, l)} \leq V_{i}^{(k, l+1)}$ shows that $a_{l}-a_{l+1} \leq 0 \Leftrightarrow a_{l+1} \geq a_{l}$ and so $\left\{a_{l}\right\}$ is a monotonically increasing sequence. Additionally, $\left\{a_{l}\right\}$ is bounded from above by the value of the corresponding storage function. Therefore, the sequence $\left\{a_{l}\right\}$ converges to a limit. Note that the equality holds if the sequence $\left\{a_{l}\right\}$ has converged To show this, let $n$ be the first time for which $a_{n-1}=a_{n}$, then one has $v_{i}^{(n)}=v_{i}^{(n+1)}$ based on (31). Moreover, $a_{n}=a_{n+1}$ according to (36). This, on the other hand, based on (31) implies that $v_{i}^{(n+1)}=v_{i}^{(n+2)}$ and consequently $a_{n+1}=a_{n+2}$. Similarly, one can show that $a_{n+i}=a_{n+i+1}$ $\forall i \geq 0$ and thus the convergence is achieved. This completes the proof of convergence for the inner-loop.

To prove the convergence of the outer-loop, integrating $\dot{V}_{i}^{(k, l)}-\dot{V}_{i}^{(k+1, l)}$ along the solution of the error system gives

$$
\begin{aligned}
\dot{\delta}_{i}= & A \delta_{i}+\left(d_{i}+g_{i}\right) B_{i} u_{i}^{k+1}-\sum_{j \in \mathrm{N}_{i}} e_{i j} B_{j} u_{j} \\
& +\left(d_{i}+g_{i}\right) D_{i} v_{i}^{l}-\sum_{j \in \mathrm{N}_{i}} e_{i j} D_{j} v_{j}
\end{aligned}
$$

and considering that only agent $i$ updates her policy, one has

$$
\begin{aligned}
& V_{i}^{(k, l)}-V_{i}^{(k+1, l)}=\int_{t}^{\infty}\left(( \frac { \partial V _ { i } ^ { ( k , l + 1 ) } } { \partial \delta _ { i } } ) ^ { T } \left(A \delta_{i}+\left(d_{i}+g_{i}\right) B_{i} u_{i}^{k+1}\right.\right. \\
& \left.-\sum_{j \in \mathrm{N}_{i}} e_{i j} B_{j} u_{j}+\left(d_{i}+g_{i}\right) D_{i} v_{i}^{l}-\sum_{j \in \mathrm{N}_{i}} e_{i j} D_{j} v_{j}\right)
\end{aligned}
$$




$$
\begin{aligned}
& -\left(\frac{\partial V_{i}^{(k, l)}}{\partial \delta_{i}}\right)^{T}\left(A \delta_{i}+\left(d_{i}+g_{i}\right) B_{i} u_{i}^{k+1}-\sum_{j \in \mathrm{N}_{i}} e_{i j} B_{j} u_{j}\right. \\
& \left.\left.+\left(d_{i}+g_{i}\right) D_{i} v_{i}^{l}-\sum_{j \in \mathrm{N}_{i}} e_{i j} D_{j} v_{j}\right)\right) d t
\end{aligned}
$$

Using (30) for the first and second terms of the right-hand side of the above equation one has

$$
\begin{aligned}
& V_{i}^{(k, l)}-V_{i}^{(k+1, l)}=\int_{t}^{\infty}\left(\frac{1}{2} u_{i}^{k T} R_{i i} u_{i}^{k}-\frac{1}{2} u_{i}^{(k+1) T} R_{i i} u_{i}^{(k+1)}+\right. \\
& \left.\left(\frac{\partial V_{i}^{(k, l)}}{\partial \delta_{i}}\right)^{T}\left(d_{i}+g_{i}\right) B_{i} u_{i}^{k}-\left(\frac{\partial V_{i}^{(k, l)}}{\partial \delta_{i}}\right)^{T}\left(d_{i}+g_{i}\right) B_{i} u_{i}^{k+1}\right) d t
\end{aligned}
$$

Then, substituting (32) into (39) and doing some manipulations gives

$$
V_{i}^{(k, l)}-V_{i}^{(k+1, l)}=\int_{t}^{\infty}\left(\frac{1}{2}\left(u_{i}^{(k+1)}-u_{i}^{k}\right)^{T} R_{i i}\left(u_{i}^{(k+1)}-u_{i}^{k}\right)\right) d t \geq 0
$$

Therefore, $V_{i}^{(k, l)} \geq V_{i}^{(k+1, l)}$. Defining the sequence $a_{k}=V_{i}^{(k, l)}$, the inequality $V_{i}^{(k, l)} \geq V_{i}^{(k+1, l)}$ shows that $a_{k} \geq a_{k+1}$. Therefore, the sequence $\left\{a_{k}\right\}$ is monotonically decreasing and bounded from below, and so it converges to a limit. Note that the equality holds if the sequence $\left\{a_{k}\right\}$ has converged. To show this, Let $m$ be the first time for which $a_{m-1}=a_{m}$, then one has $u_{i}^{(m)}=u_{i}^{(m+1)}$ based on (32) Moreover, $a_{m}=a_{m+1}$ according to (40). This, on the other hand, based on (32) implies that $u_{i}^{(m+1)}=u_{i}^{(m+2)}$ and consequently $a_{m+1}=a_{m+2}$. Similarly, one can show that $a_{m+i}=a_{m+i+1}, \forall i \geq 0$ and thus the convergence is achieved. This completes the proof of convergence for the inner-loop.

Consequently, according to the above results and the principle of optimality, one can conclude that the algorithm converges to $V_{i}^{*}$, the solution to the best response HJI equation (29).

The next theorem proves the convergence of Algorithm 1 for the case where all agents update their policies simultaneously. Define the relative weights $\rho_{i j}=\bar{\sigma}\left(R_{j j}^{-1} R_{i j}\right)$, and $\beta_{i j}=\bar{\sigma}\left(T_{i j}^{-1} T_{i j}\right)$.

Theorem 4. Assume that the policies in the neighborhood are updated and that there exist a stabilizing control policy and disturbance policy with $\gamma \geq \gamma^{*}$. Assume that agent $i$ performs Algorithm 1. Then, for small weights $e_{i j}$, and relative weights $\beta_{i j}, \rho_{i j}$, the policies $u_{i}, u_{i}, v_{i}, v_{i}$ converge to the Nash equilibrium, and the game values converge to $V_{i}^{(k, l)} \rightarrow V_{i}^{*}$, which $V_{i}^{*}$ are the solutions to the HJI equations (18).

Proof. Firstly, we prove the convergence of the inner-loop. By integrating $\dot{V}_{i}^{(k, l)}-\dot{V}_{i}^{(k, l+1)}$ along the solution of the error system

$$
\begin{aligned}
\dot{\delta}_{i}= & A \delta_{i}+\left(d_{i}+g_{i}\right) B_{i} u_{i}^{k}-\sum_{j \in \mathrm{N}_{i}} e_{i j} B_{j} u_{j}^{k} \\
& +\left(d_{i}+g_{i}\right) D_{i} v_{i}^{l+1}-\sum_{j \in \mathrm{N}_{i}} e_{i j} D_{j} v_{j}^{l}
\end{aligned}
$$

and considering that all agents update their policies, one has

$$
\begin{aligned}
& V_{i}^{(k, l)}-V_{i}^{(k, l+1)}=\int_{t}^{\infty}\left(( \frac { \partial V _ { i } ^ { ( k , l + 1 ) } } { \partial \delta _ { i } } ) ^ { T } \left(A \delta_{i}+\left(d_{i}+g_{i}\right) B_{i} u_{i}^{k}\right.\right. \\
& \left.-\sum_{j \in \mathrm{N}_{i}} e_{i j} B_{j} u_{j}^{k}+\left(d_{i}+g_{i}\right) D_{i} v_{i}^{l+1}-\sum_{j \in \mathrm{N}_{i}} e_{i j} D_{j} v_{j}^{l}\right) \\
& -\left(\frac{\partial V_{i}^{(k, l)}}{\partial \delta_{i}}\right)^{T}\left(A \delta_{i}+\left(d_{i}+g_{i}\right) B_{i} u_{i}^{k}-\sum_{j \in \mathrm{N}_{i}} e_{i j} B_{j} u_{j}^{k}\right. \\
& \left.\left.+\left(d_{i}+g_{i}\right) D_{i} v_{i}^{l+1}-\sum_{j \in \mathrm{N}_{i}} e_{i j} D_{j} v_{j}^{l}\right)\right) d t
\end{aligned}
$$

Using (30) for the first and second the above equation yields

$$
\begin{aligned}
& V_{i}^{(k, l)}-V_{i}^{(k, l+1)}=\int_{t}^{\infty}\left(\frac{1}{2} \gamma^{2} v_{i}^{(l+1) T} T_{i i} v_{i}^{(l+1)}+\frac{1}{2} \gamma^{2} \sum_{j \in N_{i}} v_{j}^{(l+1) T} T_{i j} v_{j}^{(l+1)}\right. \\
& +\left(\frac{\partial V_{i}^{(k, l+1)}}{\partial \delta_{i}}\right)^{T} \sum_{j \in \mathrm{N}_{i}} e_{i j} D_{j} v_{j}^{l+1}-\left(\frac{\partial V_{i}^{(k, l+1)}}{\partial \delta_{i}}\right)^{T} \sum_{j \in \mathrm{N}_{i}} e_{i j} D_{j} v_{j}^{l} \\
& -\frac{1}{2} \gamma^{2} v_{i}^{l T} T_{i i} v_{i}^{l}-\frac{1}{2} \gamma^{2} \sum_{j \in N_{i}} v_{j}^{l T} T_{i j} v_{j}^{l}+\left(\frac{\partial V_{i}^{(k, l)}}{\partial \delta_{i}}\right)^{T}\left(d_{i}+g_{i}\right) D_{i} v_{i}^{l} \\
& \left.-\left(\frac{\partial V_{i}^{(k, l)}}{\partial \delta_{i}}\right)^{T}\left(d_{i}+g_{i}\right) D_{i} v_{i}^{l+1}\right) d t
\end{aligned}
$$

Then, substituting (31) into (43) and doing some manipulations gives

$$
\begin{aligned}
& V_{i}^{(k, l)}-V_{i}^{(k, l+1)}=\int_{t}^{\infty}\left(-\frac{1}{2} \gamma^{2}\left(v_{i}^{l+1}-v_{i}^{l}\right)^{T} T_{i i}\left(v_{i}^{l+1}-v_{i}^{l}\right)\right. \\
& -\frac{1}{2} \gamma^{2} \sum_{j \in \mathrm{N}_{i}}\left(v_{j}^{l+1}-v_{j}^{l}\right)^{T} T_{i j}\left(v_{j}^{l+1}-v_{j}^{l}\right)+\gamma^{2} \sum_{j \in \mathrm{N}_{i}} v_{j}^{(l+1) T} T_{i j} \times \\
& \left.\left(v_{j}^{l+1}-v_{j}^{l}\right)+\sum_{j \in \mathrm{N}_{i}}\left(\frac{\partial V_{i}^{(k, l+1)}}{\partial \delta_{i}}\right)^{T} e_{i j} D_{j}\left(v_{j}^{l+1}-v_{j}^{l}\right)\right) d t
\end{aligned}
$$

A sufficient condition for $V_{i}^{(k, l)} \leq V_{i}^{(k, l+1)}$ is

$$
\begin{aligned}
& \frac{1}{2} \gamma^{2} \underline{\sigma}\left(T_{i j}\right)\left\|\Delta v_{j}\right\|>\left(d_{j}+g_{j}\right) \beta_{i j}\left\|\frac{\partial V_{j}^{(k, l)}}{\partial \delta_{j}}\right\| \cdot\left\|D_{j}\right\| \\
& +e_{i j}\left\|\frac{\partial V_{i}^{(k, l+1)}}{\partial \delta_{i}}\right\| \cdot\left\|D_{j}\right\|
\end{aligned}
$$

where $\Delta v_{j}=v_{j}^{l+1}-v_{i}^{l}$, and $\sigma\left(T_{i j}\right)$ is the minimum singular value of $T_{i j}$. This holds if $e_{i j}=0, \beta_{i j}=0$. By continuity, $V_{i}^{(k, l)} \leq V_{i}^{(k, l+1)}$ holds for small values of $e_{i i}, \beta_{i j}$. Similar to Theorem 3, one can show that the equality holds if the sequence has converged, and this completes the proof.

We now prove the convergence of the outer loop. By integrating $\dot{V}_{i}^{(k, l)}-\dot{V}_{i}^{(k+1, l)}$ along the solution of

$$
\begin{aligned}
\dot{\delta}_{i}= & A \delta_{i}+\left(d_{i}+g_{i}\right) B_{i} u_{i}^{k+1}-\sum_{j \in \mathrm{N}_{i}} e_{i j} B_{j} u_{j}^{k} \\
& +\left(d_{i}+g_{i}\right) D_{i} v_{i}^{l}-\sum_{j \in \mathrm{N}_{i}} e_{i j} D_{j} v_{j}^{l}
\end{aligned}
$$

and considering that all agents update their policies, one has 


$$
\begin{aligned}
& V_{i}^{(k, l)}-V_{i}^{(k+1, l)}=\int_{t}^{\infty}\left(( \frac { \partial V _ { i } ^ { ( k + 1 , l ) } } { \partial \delta _ { i } } ) ^ { T } \left(A \delta_{i}+\left(d_{i}+g_{i}\right) B_{i} u_{i}^{k+1}\right.\right. \\
& \left.-\sum_{j \in \mathrm{N}_{i}} e_{i j} B_{j} u_{j}^{k}+\left(d_{i}+g_{i}\right) D_{i} v_{i}^{l}-\sum_{j \in \mathrm{N}_{i}} e_{i j} D_{j} v_{j}^{l}\right) \\
& -\left(\frac{\partial V_{i}^{(k, l)}}{\partial \delta_{i}}\right)^{T}\left(A \delta_{i}+\left(d_{i}+g_{i}\right) B_{i} u_{i}^{k+1}-\sum_{j \in \mathrm{N}_{i}} e_{i j} B_{j} u_{j}^{k}\right. \\
& \left.\left.+\left(d_{i}+g_{i}\right) D_{i} v_{i}^{l}-\sum_{j \in \mathrm{N}_{i}} e_{i j} D_{j} v_{j}^{l}\right)\right) d t
\end{aligned}
$$

Using (30), this equation becomes

$$
\begin{aligned}
& V_{i}^{(k, l)}-V_{i}^{(k+1, l)}=\int_{t}^{\infty}\left(\frac{1}{2} u_{i}^{k T} R_{i i} u_{i}^{k}+\frac{1}{2} \sum_{j \in \mathrm{N}_{i}} u_{j}^{k T} R_{i j} u_{j}^{k}\right. \\
& +\left(\frac{\partial V_{i}^{(k, l)}}{\partial \delta_{i}}\right)^{T}\left(d_{i}+g_{i}\right) B_{i} u_{i}^{k}-\left(\frac{\partial V_{i}^{(k, l)}}{\partial \delta_{i}}\right)^{T}\left(d_{i}+g_{i}\right) B_{i} u_{i}^{k+1} \\
& -\frac{1}{2} u_{i}^{(k+1) T} R_{i i} u_{i}^{k+1}-\frac{1}{2} \sum_{j \in \mathrm{N}_{i}} u_{j}^{(k+1) T} R_{i j} u_{j}^{k+1}+\left(\frac{\partial V_{i}^{(k+1, l)}}{\partial \delta_{i}}\right)^{T} \times \\
& \left.\sum_{j \in \mathrm{N}_{i}} e_{i j} B_{j} u_{j}^{k+1}-\left(\frac{\partial V_{i}^{(k+1, l)}}{\partial \delta_{i}}\right)^{T} \sum_{j \in \mathrm{N}_{i}} e_{i j} B_{j} u_{j}^{k}\right) d t
\end{aligned}
$$

Then, substituting (32) into (48) and doing some manipulations gives

$$
\begin{aligned}
& V_{i}^{(k, l)}-V_{i}^{(k+1, l)}=\int_{t}^{\infty}\left(\frac{1}{2}\left(u_{i}^{k+1}-u_{i}^{k}\right)^{T} R_{i i}\left(u_{i}^{k+1}-u_{i}^{k}\right)\right. \\
& +\frac{1}{2} \sum_{j \in \mathrm{N}_{i}}\left(u_{j}^{k+1}-u_{j}^{k}\right)^{T} R_{i j}\left(u_{j}^{k+!}-u_{j}^{k}\right)-\sum_{j \in \mathrm{N}_{i}} u_{j}^{(k+1) T} R_{i j} \times \\
& \left.\left(u_{j}^{k+1}-u_{j}^{k}\right)+\left(\frac{\partial V_{i}^{(k+1, l)}}{\partial \delta_{i}}\right)^{T} \sum_{j \in \mathrm{N}_{i}} e_{i j} B_{j}\left(u_{j}^{k+1}-u_{j}^{k}\right)\right) d t
\end{aligned}
$$

A sufficient condition for $V_{i}^{(k, l)} \geq V_{i}^{(k+1, l)}$ is

$$
\begin{aligned}
& \frac{1}{2} \underline{\sigma}\left(R_{i j}\right)\left\|\Delta u_{j}\right\|>\left(d_{j}+g_{j}\right) \rho_{i j}\left\|\frac{\partial V_{j}^{(k, l)}}{\partial \delta_{j}}\right\| \cdot\left\|B_{j}\right\| \\
& +e_{i j}\left\|\frac{\partial V_{i}^{(k+1, l)}}{\partial \delta_{i}}\right\| \cdot\left\|B_{j}\right\|
\end{aligned}
$$

where $\Delta u_{j}=u_{j}^{k+1}-u_{j}^{k}$, and $\underline{\sigma}\left(R_{i j}\right)$ is the minimum singular value of $R_{i j}$. This holds if $e_{i j}=0, \rho_{i j}=0$. By continuity, $V_{i}^{(k, l)} \geq V_{i}^{(k+1, l)}$ holds for small values of $e_{i j}, \rho_{i j}$. Similar to Theorem 3, one can show that the equality holds if the sequence has converged, and this completes the proof.

Consequently, according to the above results and the principle of optimality we can say that the algorithm converges to $V_{i}^{*}$, the solution to the coupled HJI equation (18).

Remark 3. Note that Algorithm 1 converges even for the asynchronous update case, in which each agent updates her policy independently. To show this, let us divide the neighbors of the agent $i$ into two groups: those who update their policies (denoted by $\mathrm{N}_{i 1}$ ) and those who have fixed policies (denoted by $\mathrm{N}_{i 2}$ ). Then, using the same procedure as the proof of Theorem 3 and 4, one can get

$$
\begin{aligned}
& V_{i}^{(k, l)}-V_{i}^{(k, l+1)}=\int_{t}^{\infty}\left(-\frac{1}{2} \gamma^{2}\left(v_{i}^{l+1}-v_{i}^{l}\right)^{T} T_{i i}\left(v_{i}^{l+1}-v_{i}^{l}\right)\right. \\
& -\frac{1}{2} \gamma^{2} \sum_{j_{2} \in \mathrm{N}_{i 2}}\left(v_{j_{2}}^{l+1}-v_{j_{2}}^{l}\right)^{T} T_{i j_{2}}\left(v_{j_{2}}^{l+1}-v_{j_{2}}^{l}\right)+\gamma^{2} \sum_{j_{2} \in \mathrm{N}_{i 2}} v_{j_{2}}^{(l+1) T} T_{i j_{2}} \times \\
& \left.\left(v_{j_{2}}^{l+1}-v_{j_{2}}^{l}\right)+\sum_{j_{2} \in \mathrm{N}_{i 2}}\left(\frac{\partial V_{i}^{(k, l+1)}}{\partial \delta_{i}}\right)^{T} e_{i j_{2}} D_{j_{2}}\left(v_{j_{2}}^{l+1}-v_{j_{2}}^{l}\right)\right) d t
\end{aligned}
$$

The difference between this equation and (44) is that in (44) the sums are over all neighbors of agent $i$, while for this equation, the sums are only over those neighbors of agent $i$ who update their policies. Therefore, the rest of the proof of the convergence of Algorithm 1 for the synchronous update case is the same as the proof of Theorem 4.

\section{Online Policy Iteration for Learning the Solution to the HJI equations in Real Time}

In this section, the solution to the HJI equations (18) is learned online in real time based on policy iteration Algorithm 1. An actor-critic-disturbance architecture is used to approximate the solution to these HJI equations (Abu-Khalaf, Lewis, \& Huang, 2006, 2008; Modares, Lewis, \& Naghibi-Sistani, 2014; Vamvoudakis, \& Lewis, 2012). Specifically, each agent has three neural networks (NNs): one critic, one control actor and one disturbance. That is, for $N$ agents, $N$ critic neural networks (NNs) are used to approximate the value functions in the Bellman equations (30), $N$ actor NNs are used to approximate the control policies (32) and $N$ disturbance NNs are used to approximate the disturbance policies (31). All these $3 \mathrm{~N}$ NNs are tuned simultaneously and in real time.

\subsection{Critic neural network}

According to the Weierstrass higher-order approximation Theorem (Abou-Khalaf \& Lewis, 2005), there exist NNs such that the smooth value functions $V_{i}$ are approximated using critic NNs as

$$
V_{i}\left(\delta_{i}\right)=W_{i}^{T} \phi_{i}\left(\delta_{i}\right)+\varepsilon_{i}
$$

where $W_{i}$ are the critic NN weights, $\phi_{i} \in R^{h}$ are the critic $\mathrm{NN}$ activation function vectors, with $h$ the number of neurons in the hidden layer of the critic NNs. According to the Weierstrass Theorem, the NN approximation error $\varepsilon_{i}$ converges to zero uniformly as $h \rightarrow \infty$. Assuming current weight estimates $\hat{W}_{i}$, the outputs of the critic NNs are given by

$$
\hat{V}_{i}=\hat{W}_{i}^{T} \phi_{i}
$$

Then, the Bellman equation (30) can be approximated at each step as 


$$
\begin{aligned}
& H_{i}\left(\delta_{i}, \hat{W}_{i}, u_{i}, u_{-i}\right)=\frac{1}{2} \delta_{i}^{T} Q_{i i} \delta_{i}+\frac{1}{2} u_{i}^{T} R_{i i} u_{i}+\frac{1}{2} \sum_{j \in N_{i}} u_{j}^{T} R_{i j} u_{j} \\
& -\frac{1}{2} \gamma^{2} v_{i}^{T} T_{i i} v_{i}-\frac{1}{2} \gamma^{2} \sum_{j \in \mathrm{N}_{i}} v_{j}^{T} T_{i j} v_{j}+\hat{W}_{i}^{T} \frac{\partial \phi_{i}}{\partial \delta_{i}}\left(A \delta_{i}+\left(d_{i}+g_{i}\right) B_{i} u_{i}\right. \\
& \left.-\sum_{j \in N_{i}} e_{i j} B_{j} u_{j}+\left(d_{i}+g_{i}\right) D_{i} v_{i}-\sum_{j \in \mathrm{N}_{i}} e_{i j} D_{j} v_{j}\right)=e_{H_{i}}
\end{aligned}
$$

It is desired to select $\hat{W}_{i}$ to minimize the square residual error

$$
E_{1}=\frac{1}{2} e_{H_{i}}^{2}
$$

Then $\hat{W}_{i} \rightarrow W_{i}$ which solves (53) in a least-squares sense and $e_{H_{i}}$ becomes small. Theorem 5 gives a tuning law for the critic weights that minimizes (54).

\subsection{Actor and disturbance neural networks}

Based on the control update policies (16), the control policies are given as actor neural networks (Vamvoudakis, Lewis, \& Hudas 2012) as follows,

$$
\hat{u}_{i} \equiv \hat{u}_{i+N}=-\left(d_{i}+g_{i}\right) R_{i i}^{-1} B_{i}^{T} \frac{\partial \phi_{i}{ }^{T}}{\partial \delta_{i}} \hat{W}_{i+N}
$$

where $\hat{W}_{i+N}$ denote the current estimated values of the ideal critic NN weights $W_{i}$. Moreover, based on the disturbance update policies (17), the disturbance neural networks are chosen as

$$
\hat{v}_{i} \equiv \hat{v}_{i+2 N}=\frac{1}{\gamma^{2}}\left(d_{i}+g_{i}\right) T_{i i}^{-1} D_{i}^{T} \frac{\partial \phi_{i}^{T}}{\partial \delta_{i}} \hat{W}_{i+2 N}
$$

where $\hat{W}_{i+2 N}$ denote the current estimated values of the ideal critic NN weights $W_{i}$. The notation $\hat{u}_{i+N}$ and $\hat{v}_{i+2 N}$ in (55) and (56) are used to keep indices straight in the proof (the first $N$ weights are used for the critics, the second $N$ weights are for the actors and the rest for the disturbances).

The following two theorems provide the tuning laws for the actor, critic and disturbance NNs that guarantee convergence of the synchronous online multiple agent learning PI algorithm in real time to the game saddle point solution, while guaranteeing closed-loop stability. Define the critic, actor and disturbance NN estimation errors as $\tilde{W}_{i}=W_{i}-\hat{W}_{i}, \tilde{W}_{i+N}=W_{i}-\hat{W}_{i+N}$ and $\tilde{W}_{i+2 N}=W_{i}-\hat{W}_{i+2 N}$.

Theorem 5. (Online zero-sum graphical games) Let the error dynamics be given by (8), and consider the zero-sum differential graphical game formulation in (11). Let the critic NN at each node be given by (52), the control input be given by actor NN (55), and the disturbance input be given by disturbance NN (56). Let tuning for the $i$ th critic $\mathrm{NN}$ be provided by

$$
\begin{aligned}
& \dot{\hat{W}}_{i}=-a_{i} \frac{\partial E_{1}}{\partial \hat{W}_{i}}=-a_{i} \frac{\sigma_{i+N}}{\left(1+\sigma_{i+N}{ }^{\mathrm{T}} \sigma_{i+N}\right)^{2}}\left[\sigma_{i+N}{ }^{\mathrm{T}} \hat{W}_{i}+\frac{1}{2} \delta_{i}^{T} Q_{i i} \delta_{i}\right. \\
& +\frac{1}{2}\left(d_{i}+g_{i}\right)^{2} \hat{W}_{i+N}{ }^{T} \bar{D}_{i} \hat{W}_{i+N}-\frac{1}{2 \gamma^{2}}\left(d_{i}+g_{i}\right)^{2} \hat{W}_{i+2 N}{ }^{T} \bar{E}_{i} \hat{W}_{i+2 N}
\end{aligned}
$$

$$
\begin{aligned}
& +\frac{1}{2} \sum_{j \in N_{i}}\left(d_{j}+g_{j}\right)^{2} \hat{W}_{j+N}{ }^{T} \frac{\partial \phi_{j}}{\partial \delta_{j}} B_{j} R_{j j}^{-T} R_{i j} R_{j j}^{-1} B_{j}{ }^{T} \frac{\partial \phi_{j}^{T}}{\partial \delta_{j}} \hat{W}_{j+N} \\
& \left.-\frac{1}{2 \gamma^{2}} \sum_{j \in N_{i}}\left(d_{j}+g_{j}\right)^{2} \hat{W}_{j+2 N}{ }^{T} \frac{\partial \phi_{j}}{\partial \delta_{j}} D_{j} T_{j j}^{-T} T_{i j} T_{j j}^{-1} D_{j}{ }^{T} \frac{\partial \phi_{j}^{T}}{\partial \delta_{j}} \hat{W}_{j+2 N}\right]
\end{aligned}
$$

where

$$
\begin{aligned}
\sigma_{i+N}= & \frac{\partial \phi_{i}}{\partial \delta_{i}}\left(A \delta_{i}+\left(d_{i}+g_{i}\right) B_{i} \hat{u}_{i+N}-\sum_{j \in N_{i}} e_{i j} B_{j} \hat{u}_{j+N}\right. \\
& \left.+\left(d_{i}+g_{i}\right) D_{i} \hat{v}_{i+2 N}-\sum_{j \in \mathrm{N}_{i}} e_{i j} D_{j} \hat{v}_{j+2 N}\right)
\end{aligned}
$$

Let the $i$ th actor $\mathrm{NN}$ be tuned as

$$
\begin{aligned}
& \dot{\hat{W}}_{i+N}=-a_{i+N}\left\{\left(S_{i} \hat{W}_{i+N}-F_{i} \bar{\sigma}_{i+N}^{T} \hat{W}_{i}\right)-\left(d_{i}+g_{i}\right)^{2} \bar{D}_{i} \times\right. \\
& \hat{W}_{i+N} \frac{\bar{\sigma}_{i+N}{ }^{T}}{m_{s i}} \hat{W}_{i}-\sum_{j \in N_{i}}\left(d_{j}+g_{j}\right)^{2} \frac{\partial \phi_{j}}{\partial \delta_{j}} B_{j} R_{j j}^{-T} \times \\
& \left.R_{i j} R_{j j}^{-1} B_{j}{ }^{T} \frac{\partial \phi_{j}{ }^{T}}{\partial \delta_{j}} \hat{W}_{i+N} \frac{\bar{\sigma}_{i+N}{ }^{T}}{m_{s_{i}}} \hat{W}_{j}\right\}
\end{aligned}
$$

and the $i$ th disturbance $\mathrm{NN}$ be tuned as

$$
\begin{aligned}
& \dot{\hat{W}}_{i+2 N}=-a_{i+2 N}\left\{\left(\bar{S}_{i} \hat{W}_{i+2 N}-\bar{F}_{i} \bar{\sigma}_{i+N}^{T} \hat{W}_{i}\right)+\frac{1}{\gamma^{2}}\left(d_{i}+g_{i}\right)^{2} \times\right. \\
& \bar{E}_{i} \hat{W}_{i+2 N} \frac{\bar{\sigma}_{i+N}{ }^{T}}{m_{s i}} \hat{W}_{i}+\frac{1}{\gamma^{2}} \sum_{j \in N_{i}}\left(d_{j}+g_{j}\right)^{2} \frac{\partial \phi_{j}}{\partial \delta_{j}} D_{j} T_{j j}^{-T} \times \\
& \left.T_{i j} T_{j j}^{-1} D_{j}^{T} \frac{\partial \phi_{j}^{T}}{\partial \delta_{j}} \hat{W}_{i+2 N} \frac{\bar{\sigma}_{i+N}{ }^{T}}{m_{s_{i}}} \hat{W}_{j}\right\}
\end{aligned}
$$

where

$\bar{D}_{i}(x) \equiv \frac{\partial \phi_{i}}{\partial \delta_{i}} B_{i} R_{i i}^{-1} B_{i}^{T} \frac{\partial \phi_{i}{ }^{T}}{\partial \delta_{i}}, \bar{E}_{i}(x) \equiv \frac{\partial \phi_{i}}{\partial \delta_{i}} D_{i} T_{i i}^{-1} D_{i}^{T} \frac{\partial \phi_{i}{ }^{T}}{\partial \delta_{i}}$,

and $m_{s_{i}} \equiv\left(\sigma_{i+N}{ }^{T} \sigma_{i+N}+1\right), \bar{\sigma}_{i+N}=\sigma_{i+N} /\left(\sigma_{i+N}{ }^{T} \sigma_{i+N}+1\right)$ and $a_{i}>0, \ldots a_{i+N}>0, \ldots a_{i+2 N}>0 \quad, \quad F_{i}>0, S_{i}>0, \bar{F}_{i}>0$, $\vec{S}_{i}>0, \quad i \in N$ are tuning parameters. Suppose that $\bar{\sigma}_{i+N}=\sigma_{i+N} /\left(\sigma_{i+N}{ }^{T} \sigma_{i+N}+1\right)$ is persistently exciting. Then the closed-loop system states $\delta_{i}(t)$, the critic NN errors $\tilde{W}_{i}$, the actor NN errors $\tilde{W}_{i+N}$ and the disturbance NN errors $\tilde{W}_{i+2 N}$ are uniformly ultimately bounded.

Proof: The proof is similar to (Vamvoudakis, Lewis, \& Hudas, 2012; Vamvoudakis, \& Lewis, 2012) and is omitted.

Theorem 6. (Convergence to zero-sum Nash equilibrium) Suppose the hypotheses of Theorem 5 hold. Then:

a) $H_{i}\left(\delta_{i}, \hat{W}_{i}, \hat{u}_{i}, \hat{u}_{-i}, \hat{v}_{i}, \hat{v}_{-i}\right), \forall i \in N \quad$ are uniformly ultimately bounded, that is, $\hat{W}_{i}$ converge to the approximate coupled HJI solution, the value of the ZS game, where

$$
\hat{u}_{i}=-\left(d_{i}+g_{i}\right) R_{i i}^{-1} B_{i}^{T} \frac{\partial \phi_{i}^{T}}{\partial \delta_{i}} \hat{W}_{i}, \hat{v}_{i}=\frac{1}{\gamma^{2}}\left(d_{i}+g_{i}\right) T_{i i}^{-1} D_{i}^{T} \frac{\partial \phi_{i}^{T}}{\partial \delta_{i}} \hat{W}_{i}
$$


b) $\hat{u}_{i+N}, \hat{v}_{i+2 N}$ (see (55) and (56)) converge to the approximate Nash equilibrium solution of the zero-sum game.

Proof: The proof is similar to (Vamvoudakis, Lewis, \& Hudas 2012 ; Vamvoudakis, \& Lewis, 2012) and is omitted.

\section{Simulation Results}

In this section, the effectiveness of the online approach described in Theorem 5 is shown by a simulation example.

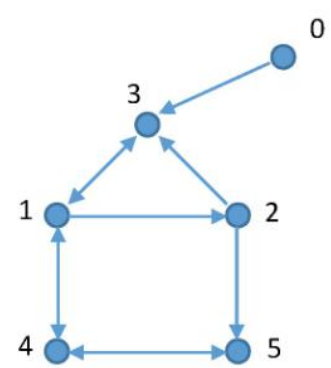

Fig. 1. Communication Graph.

For the digraph structure (Fig.1) shown in Vamvoudakis, Lewis, and Hudas (2012), we consider the following node dynamics

$$
\dot{x}_{i}=A x_{i}+B_{i} u_{i}+D_{i} v_{i}
$$

where $A=\left[\begin{array}{cc}0 & 0.1 \\ -0.1 & 0\end{array}\right], B_{1}=\left[\begin{array}{c}2 \\ -1\end{array}\right], B_{2}=\left[\begin{array}{c}2 \\ -3\end{array}\right], B_{3}=\left[\begin{array}{c}2 \\ -1\end{array}\right]$, $B_{4}=\left[\begin{array}{c}1 \\ -3\end{array}\right], \quad B_{5}=\left[\begin{array}{c}1 \\ -2\end{array}\right], \quad D_{1}=\left[\begin{array}{c}2 \\ -1\end{array}\right], \quad D_{2}=\left[\begin{array}{c}2 \\ -2\end{array}\right]$, $D_{3}=\left[\begin{array}{c}2 \\ -2\end{array}\right], D_{4}=\left[\begin{array}{c}2 \\ -3\end{array}\right], D_{5}=\left[\begin{array}{c}2 \\ -2\end{array}\right]$,

with target generator $\dot{x}_{0}=A x_{0}$.

The graphical game is implemented as in Theorem 5 . Persistence of excitation was ensured by adding a small exponentially decreasing probing noise to the control inputs. The dynamics of each agent is marginally stable and so synchronization is required to a Lissajous pattern in the phase plane. Figs. 2 and 3 show the evolution of the system for agents $1,2,3,4,5$ and leader 0 . Fig. 4 shows the 3-D phase plane plot of all five agents and the leader node.

\section{Conclusion}

This paper provides a new perspective on the synchronization of multi-agent linear systems influenced by external disturbances. It brings together distributed control, optimal control theory and differential game theory to formulate and solve the multi-player zero-sum graphical games. It is shown that the solution to the given problem requires the solution to coupled HJI equations. It is also shown that the HJI solution makes the global synchronization error $\mathrm{L}_{2}$-bounded. A policy iteration algorithm is presented to solve coupled HJI equations and her conver- gence to the Nash equilibrium solution of the multi-player zero-sum game is shown. An online policy iteration algorithm is given which learns the solution to these HJI equations in real time. Simulation results are provided to show the effectiveness of the proposed method.

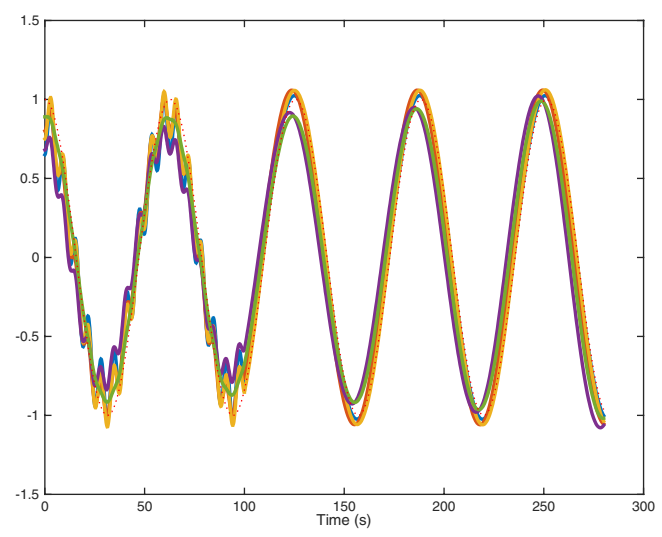

Fig. 2. Evolution of the states for $\mathrm{x} 1_{1}, \mathrm{x} 2_{1}, \mathrm{x} 3_{1}, \mathrm{x} 4_{1}, \mathrm{x} 5_{1}, \mathrm{x} 0_{1}$.

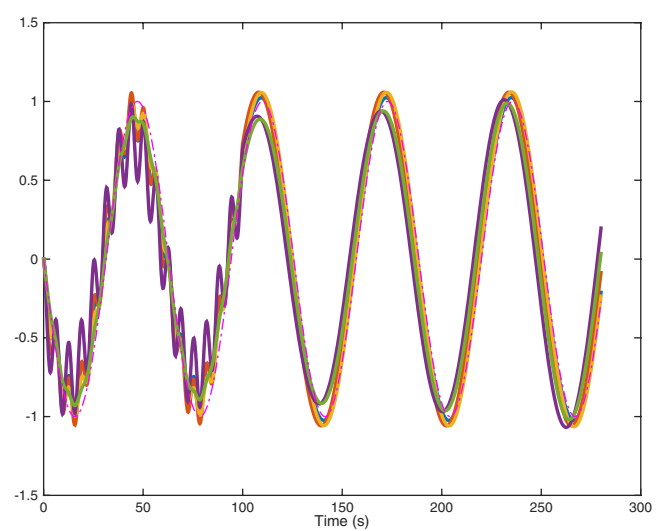

Fig. 3. Evolution of the states for $\mathrm{x} 1_{2}, \mathrm{x} 2_{2}, \mathrm{x} 3_{2}, \mathrm{x} 4_{2}, \mathrm{x} 5_{2,} \mathrm{x} 0_{2}$.

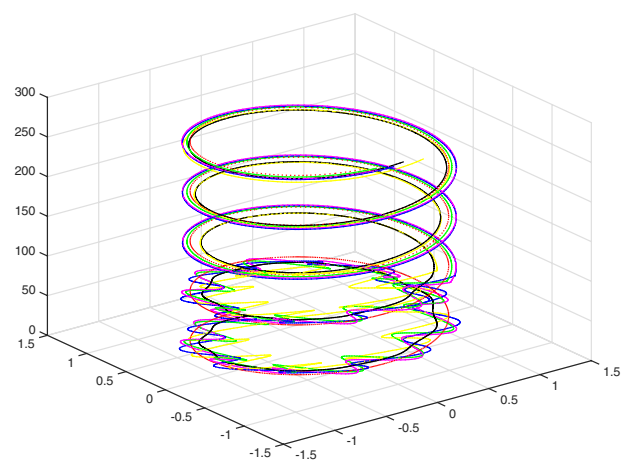

Fig. 4. 3-D Phase Plane Plot

\section{Acknowledgement}

The authors would like to acknowledge contributions to the proofs by Murad Abu-Khalaf, the executive director of 
the Kuwait-MIT Center for Natural Resources and the Environment. This work is supported by NSFC 61374087 , the Program for Changjiang Scholars and Innovative Research Team in University under Grant IRT13072, a project funded by the priority academic program development of Jiangsu Higher Education Institutions, NSF grant ECCS-1405173, ONR grant N00014-13-1-0562, ONR grant N000141410718, AFOSR EOARD Grant 13-3055, China NNSF grant 61120106011, and China Education Ministry Project 111 (No.B08015).

\section{References}

Abu-Khalaf M., \& Lewis F. L., (2005). Nearly Optimal Control Laws for Nonlinear Systems with Saturating Actuators Using a Neural Network HJB Approach, Automatica, 41(5), 779791.

Abu-Khalaf, M., Lewis F. L., \& Huang J (2006). Policy Iteration on the Hamilton-Jacobi-Isaacs Equation for $\mathrm{H} \infty$ State Feedback Control With Input Saturation. IEEE Transactions on Automatic Control, 51(12), 1989-1995.

Abu-Khalaf, M., Lewis F. L., \& Huang J (2008). Neurodynamic Programming and Zero-Sum Games for Constrained Control Systems. IEEE Transactions on Neural Networks, 19(7), $1243-1252$

Aliyu, M.D.S. (2011). Nonlinear $H_{\infty}$ control, Hamiltonian systems and Hamilton-Jacobi equations. CRC Press.

Basar, T., \& Bernhard, P. (2008). Ho Optimal Control and Related Minimax Design Problems: A Dynamic Game Approach ( $2^{\text {nd }}$ ed.). Springer.

Busoniu, L., Babuska, R., \& De Schutter, B. (2008). A Comprehensive Survey of Multiagent Reinforcement Learning. IEEE Transactions on Systems, Man, and Cybernetics, Part C: Applications and Reviews, 38(2), 156-172.

Chang, H. S. (2009). Decentralized Learning in Finite Markov Chains: Revisited. IEEE Transactions on Automatic Control, 54(7), 1648-1653.

Fax, J., \& Murray, R. (2004). Information flow and cooperative control of vehicle formations. IEEE Transactions on Automatic Control, 49(9), 1465-1476.

Hong, Y., Hu, J., \& Gao L. (2006). Tracking control for multiagent consensus with an active leader and variable topology. Automatica, 42(7), 1177-1182.

Jadbabaie, A., Lin, J., \& Morse, A. (2003). Coordination of groups of mobile autonomous agents using nearest neighbor rules. IEEE Transactions on Automatic Control, 48(6), 9881001.

Khoo, S., Xie, L., \& Man, Z. (2009). Robust Finite-Time Consensus Tracking Algorithm for Multirobot Systems. IEEE Transactions on Mechatronics, 14(2), 219-228.

Lakshmanan, H., \& Farias, D. P. (2006). Decentralized Approximate Dynamic Programming for Dynamic Networks of Agents. In Proc. Amer. control conf. (pp. 1648-1653).

Lewis, F. L., Vrabie, D. L., \& Syrmos, V. L. (2012). Optimal Control, (3rd ed.). John Wiley Sons.

Lewis, F. L., Zhang, H. W., Hengster-Movric, K., \& Das, Abhijit (2014). Cooperative control of multi-agent systems: optimal and adaptive design approaches. Berlin: Spring-Verlag.

Li X., Wang X., Chen G. (2004). Pinning a complex dynamical network to its equilibrium. IEEE Transaction on Circuitsand Systems. I. Regular Papers, 51(10), 2074-2087.

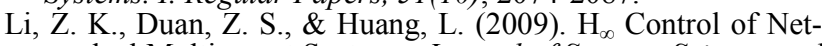
worked Multi-agent Systems. Journal of Systems Science and Complexity, 22(1), 35-48.

Li, Z. K., Duan, Z. S., \& Chen, G. R. (2011). On $\mathrm{H}_{\infty}$ and $\mathrm{H}_{2}$ performance regions of multi-agent systems. Automatica, 47(4), 797-803.

Lin, P., Jia Y. M., \& Li, L. (2008). Distributed robust $\mathrm{H}_{\infty}$ consensus control in directed networks of agents with time-delay. Systems and Control Letters, 57(8), 643-653.
Littman, M. L. (2001). Value-function reinforcement learning in Markov games. Journal of Cognitive Systems Research, 2(1), 55-66.

Liu, Y., \& Jia, Y. M. (2010). $\mathrm{H}_{\infty}$ consensus control of multiagent systems with switching topology: a dynamic output feedback control. International Journal of Control, 83(3), 527-537.

Liu, Y., \& Jia, Y. M. (2011). Robust $\mathrm{H}_{\infty}$ consensus control of uncertain multi-agent systems with time delays. International Journal of Control, Automation and Systems, 9(6), 1086-1094.

Luy, N. T., Thanh, N. T., \& Tri, H. M. (2013). Reinforcement learning-based Robust Adaptive Tracking control for Multiwheeled Mobile Robots synchronization with Optimality. IEEE Workshop on Robotic Intelligence In Informationally Structured Space, (pp. 74-81).

Modares H., Lewis F. L., \& Naghibi-Sistani M. B. (2014). Online solution of nonquadratic two-player zero-sum games arising in the $\mathrm{H}_{\infty}$ control of constrained-input systems. International Journal of Adaptive Control and Signal Processing, $28,232-254$

Olfati-Saber, R., \& Murray R. M. (2004). Consensus Problems in Networks of Agents with Switching Topology and TimeDelays. IEEE Transactions on Automatic Control, 49(9), $1520-1533$

Qu, Z. (2009). Cooperative Control of Dynamical Systems: Ap plications to Autonomous Vehicles. New York: SpringerVerlag.

Ren, W., \& Beard, R. W. (2005). Consensus seeking in multiagent systems under dynamically changing interaction topologies. IEEE Transactions on Automatic Control, 50(5), 655661.

Ren, W., \& Beard, R. W. (2008). Distributed Consensus in Multi-vehicle Cooperative Control. Berlin: Springer.

Ren, W., Beard, R. W., \& Atkins, E. M. (2005). A survey of consensus problems in multi-agent coordination. In Proc. Amer. control conf. (pp. 1859-1864).

Ren, W., Moore, K. L., Chen, Y. (2007). High-order and model reference consensus algorithms in cooperative control of multivehicle systems. Journal of Dynamic Systems, Measurement, and Control, 129(5), 678-688.

Tsitsiklis, J. (1984). Problems in decentralized decision making and computation. Ph.D. Dissertation. Dept. Elect. Eng. and Comput. Sci., MIT, Cambridge, MA

Vamvoudakis, K. G., Carrillo, L. R. G, \& Hespanha, J. P. (2013). Learning consensus in adversarial environments. SPIE Defense, Security, and Sensing. International Society for Optics and Photonics, (pp.87410K-87410K)

Vamvoudakis, K. G., \& Lewis, F. L. (2012). Online solution of nonlinear two-player zero-sum games using synchronous policy iteration. International Journal of Robust and Nonlinear Control, 22(13), 1460-1483.

Vamvoudakis, K. G., Lewis, F. L. \& Hudas, G. R. (2012). Multiagent differential graphical games: Online adaptive learning solution for synchronization with optimality. Automatica, 48(8), 1598-1611.

Vrancx, P., Verbeeck, K. \& Nowe, A. (2008). Decentralized Learning in Markov Games. IEEE Transactions on Systems, Man, and Cybernetics, Part B: Cybernetics, 38(4), 976-981.

Wang, X., \& Chen, G. (2002). Pinning control of scale-free dynamical networks. Physica A: Statistical Mechanics and its Applications, 310(3), 521-531.

Wen, G. H., Duan, Z. S., Li, Z. K., \& Chen, G. R. (2012). Consensus and its $\mathrm{L}_{2}$-gain performance of multi-agent systems with intermittent information transmissions. International Journal of Control, 85(4), 384-396.

Wheeler, Jr. R. M. \& Narendra, K. S. (1986). Decentralized learning in finite Markov chains. IEEE Transactions on Automatic Control, 31(6), 519-526.

Yang, Xi, \& Wang, J. Z. (2013). Finite-gain Lp Consensus of Multi-agent Systems. International Journal of Control, Automation and Systems 11(4), 666-674. 